DOI: https://doi.org/10.33103/uot.ijccce.20.3.3

\title{
Optimal Tuning of Linear Quadratic Regulator Controller Using Ant Colony Optimization Algorithm for Position Control of a Permanent Magnet DC Motor
}

\author{
Luay Thamir Rasheed \\ Control and Systems Engineering Department, University of Technology, Baghdad, Iraq \\ 60065@uotechnology.edu.iq
}

\begin{abstract}
This paper presents the design of an optimal Linear Quadratic Regulator (LQR) controller using Ant Colony Optimization (ACO) and particle swarm optimization (PSO) methods for position control of a permanent magnet DC (PMDC) motor. In this work, Ant Colony control and particle swarm control algorithms have been utilized to set the optimal elements of the weighting matrices subjected to a proposed cost function. The proposed cost function is a combination of the quadratic performance index and integral square error. The proposed design can overcome the difficulty in setting the weighting matrices with the suitable elements. The simulation results using (Matlab Package) show that the optimal LQR controller using ACO algorithm can give excellent performance in terms of obtaining smooth and unsaturated state voltage control action that will stabilize the DC motor system performance and minimize the position tracking error of the system output. In addition, the rising time and settling time is decreased in comparison with the LQR based PSO controller performance.
\end{abstract}

Index Terms - Linear Quadratic Regulator (LQR), Ant Colony Optimization (ACO), particle swarm optimization (PSO), quadratic performance index.

\section{INTRODUCTION}

The LQR has drawn a lot of attention since 1950 and is widely used in many applications such as flight control, missile guidance, active car suspension, power converters, ABS brake system, and etc. The LQR is an optimal multivariable feedback control method that minimizes the excursion of a system state trajectories whereas requiring minimum controller effort and improves stability performance. In other words, applying the LQR method to a controllable linear time-invariant (LTI) system results in a set of optimal feedback gains that minimize a quadratic criterion and stabilize the system [1], [2].

The behavior of the LQR controller depends on two parameters: the state and the control weighting matrices, and it must be optimally adjusted to obtain the desired performance because of their influence on the LQR performance. Moreover, the classic approaches such as trial and error, pole placement, and Bryson's method tuning LQR parameters is tedious work and time-consuming. Therefore, different optimization algorithms have implemented to obtain the optimal parameters of the LQR controller. For instance, Genetic Algorithm (GA) [3], Particle Swarm Optimization (PSO) algorithm [4], Firefly Algorithm (FFA) [5], and Artificial Bee Colony (ABC) algorithm [6], etc.

The motivation for this work is that the classic approaches in tuning the LQR parameters are still difficult. Therefore, different types of optimization algorithms have been used for 
this purpose till to date. The main points of the contribution of this paper are listed as follows:

- The ACO and PSO algorithms are used because they show fast search ability in the global regions to obtain the optimal parameters for the LQR controller. These parameters lead to finding the best voltage control action. Thus, the output position of the PMDC motor will quickly reach the desired output position.

- Excellent tracking performance of the optimal LQR controller based ACO algorithm and LQR controller based PSO algorithm that are investigated by changing the desired output position levels of the PMDC motor.

- High robustness performance was achieved by adding an external torque disturbance to the PMDC motor system using optimal LQR controllers.

The remainder of this paper is described in the following manner: Section two contains the mathematical model of the PMDC motor circuit. Section three illustrates the design structure of the optimal LQR controller. In Section four, the ACO and PSO algorithms are explained. Section five describes the performance of the optimal LQR controllers through the simulation results. Finally, the conclusions are explained in Section six.

\section{MODELLING DC MOTOR MODEL}

The PMDC motors are power actuator devices that transform electrical energy to mechanical energy and they are most suitable for adjusting and control of wide range positions and speeds. In this work, a simple model of PMDC motor was considered as depicted in Fig. 1 [7].

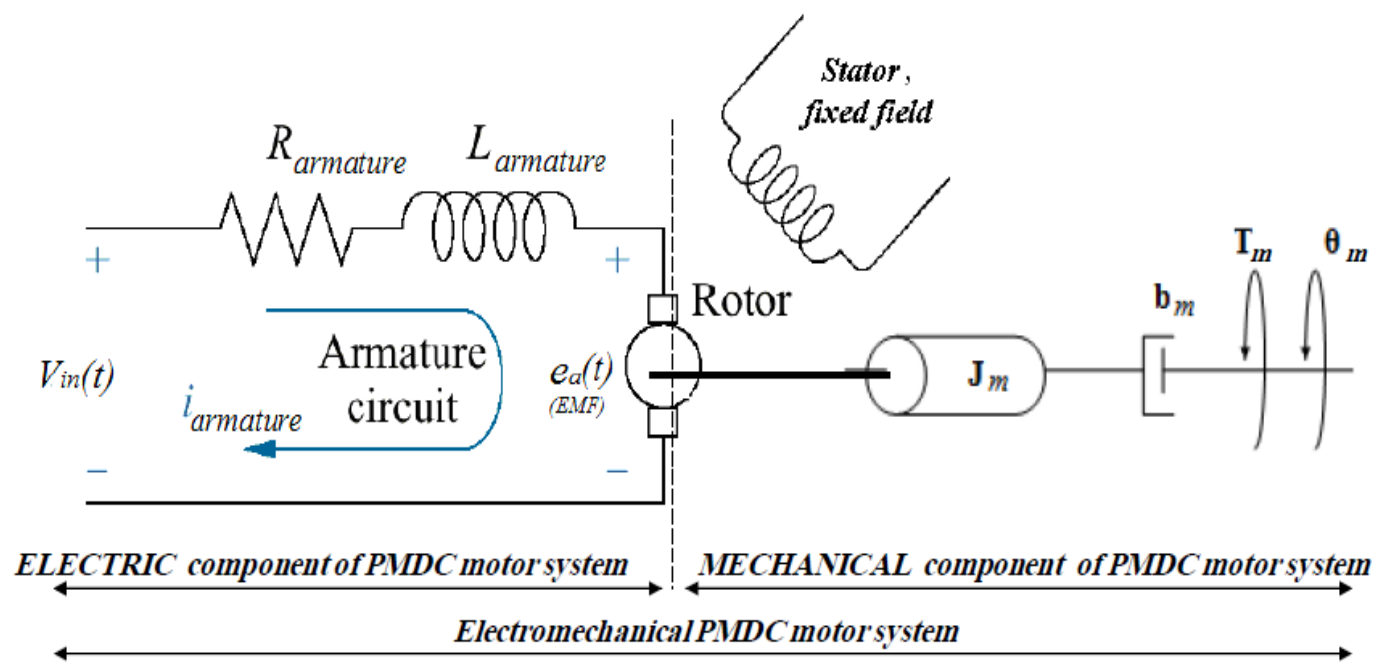

Fig. 1. A SIMPLIFIED EQUIVALENT REPRESENTATION OF THE PMDC MOTOR'S ELECTROMECHANICAL COMPONENTS [7].

In this Figure, the resistance of the armature $\left(R_{\text {armature }}\right)$ is connected in series with the inductance ( $\left.L_{\text {armature }}\right)$ and $\left(e_{a}\right)$ represents the back electromotive force (emf) voltage induced in the armature during the rotation. The armature voltage $\left(V_{i n}\right)$ in volts is driven by an input voltage source. The measured variables are the shaft angle $\left(\theta_{m}\right)$ in $\mathrm{rad}$, the angular velocity of the shaft $\left(W_{m}\right)$ in $\mathrm{rad} / \mathrm{sec}$, and the motor torque, $\left(T_{m}\right)$, in N.m. The dynamic behavior of the PMDC motor can be described by the following equations for the electrical part, mechanical part and the interconnection between them [7]- [9].

$$
\left(L_{\text {armature }} S+R_{\text {armature }}\right) I(s)=V_{\text {in }}(s)-K_{b} W_{m}(s)
$$




$$
\begin{gathered}
T_{m}(s)=K_{i} I_{\text {armature }}(s) \\
E_{a}(s)=K_{b} W_{m}(s)=K_{b} S \theta_{m}(s) \\
K_{i} I_{\text {armature }}(s)=T_{m}(s)=\left(J_{m} S+b_{m}\right) S \theta_{m}(s)+T_{d}
\end{gathered}
$$

Where, $K_{i}$ represents the torque constant, $K_{b}$ represents the emf constant, $J_{m}$ represents the moment of inertia, $b_{m}$ represents the coefficient of viscous-friction, and $T_{d}$ represents the external torque disturbance.

The block diagram for position control of PMDC motor based on equations (1), (2), (3), and (4) is depicted in Fig. 2.

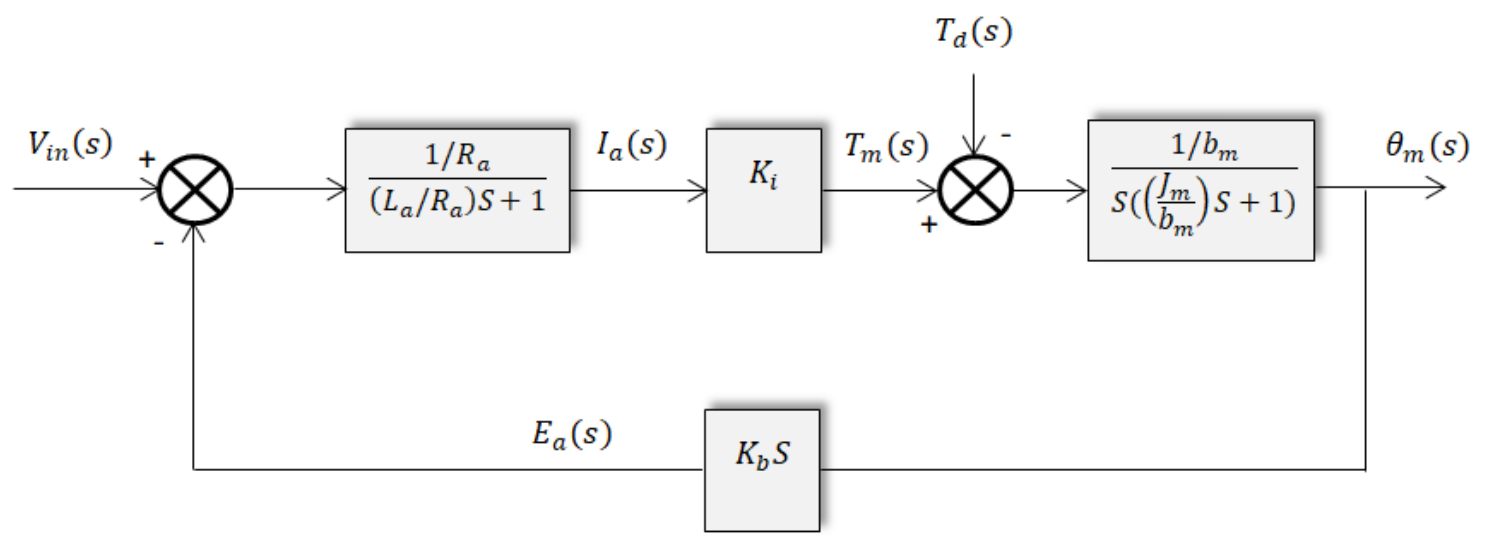

Fig. 2. THE BLOCK DIAGRAM OF THE PMDC MOTOR MODEL.

\section{OPTIMAL LQR CONTROLLER DESIGN}

The stare space representation of a linear time-invariant (LTI) system is described as follows [10]:

$$
\begin{aligned}
& \dot{\boldsymbol{x}}(t)=\boldsymbol{A} \boldsymbol{x}(t)+\boldsymbol{B} u(t) \\
& \boldsymbol{y}(t)=\boldsymbol{C} \boldsymbol{x}(t)+\boldsymbol{D} u(t)
\end{aligned}
$$

Where, $\boldsymbol{x}(t)$ is the state vector, $\boldsymbol{y}(t)$ is the output vector, and $u(t)$ is the control vector.

The linear state feedback law in LQR approach can be expressed as in equation (7):

$$
u(t)=-\boldsymbol{K} \boldsymbol{x}(t)
$$

Where, $K$ is the state feedback gain matrix.

The LQR control problem may be stated to find the optimal linear state feedback law that minimizes the quadratic performance index which is defined in equation (8):

$$
J=\int_{0}^{\infty}\left[\boldsymbol{x}(t)^{T} \boldsymbol{Q} \boldsymbol{x}(t)+u(t)^{T} \boldsymbol{R} u(t)\right] d t
$$

Where, $\boldsymbol{Q}$ is $n \times n$ symmetric positive semi definite state weighting matrix, and $\boldsymbol{R}$ is $m \times m$ symmetric positive definite control weighting matrix.

The state feedback gain matrix is given as in equation (9):

$$
K=R^{-1} B^{T} P
$$

Where, $\boldsymbol{P}$ is the unique symmetric positive semi-definite solution to the algebraic Riccati equation (ARE) which can be defined as follows:

$$
P A+A^{T} P+Q-P B R^{-1} B^{T} P=0
$$


The block diagram of this optimal LQR controller which consists of LQR controller and different types of optimization algorithm which are utilized to get the optimal values of the weighting matrices of LQR controller is shown in Fig. 3.

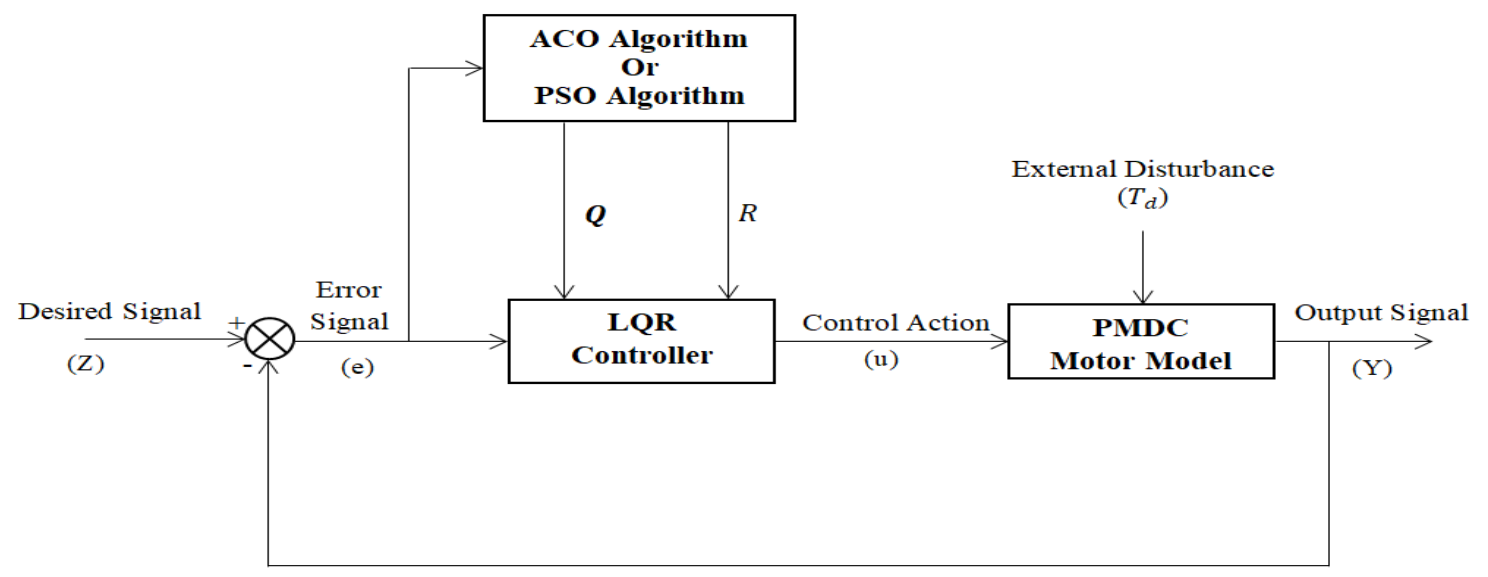

FIG. 3. THE BLOCK DIAGRAM OF TUNING LQR BASED ACO CONTROLLER.

\section{LQR TUNING USING ACO AND PSO ALGORITHMS}

\section{A. THE ACO ALGORITHM}

The ACO technique is a nature inspired meta-heuristic algorithm for the solution of the combinatorial optimization problem and was introduced by M. Dorigo in 1991.This algorithm is based on the behavior of the real ants in searching for the source of food with the minimum path cost. A graph model of the problem is formed in order to search for a minimum path cost. The ants walk along the graph to find a lower path cost and the better paths are followed after by the other ants.

Each ant releases a pheromone substance to track the graph and to select their path with probabilities that are generated because of the trails of pheromone and these pheromones are frequently decreased due to the evaporation [11]-[13]. The steps of ACO algorithm for tuning the LQR controller are given as follows:

Step 1: Initialize the algorithm parameters such as dimension of the problem (dim), population size $(N)$, maximum number of iterations (Iter), alpha $(\alpha)$, beta $(\beta)$, Evaporation rate $(\rho)$, number of nodes for each parameters $\left(n \_n o d e\right)$, pheromone Matrix $(\tau)$, and change of pheromone $(\Delta \tau)$.

Step 2: each parameter generates a number of equally spaced nodes.

Step 3: each ant calculates the probability rule to choose their solution to the problem, known as a tour as follows:

$$
\begin{gathered}
P_{i j}^{k}=\frac{\left[\tau_{i j}\right]^{\alpha}\left[\eta_{i j}\right]^{\beta}}{\sum_{i j \epsilon A^{k}}\left[\tau_{i j}\right]^{\alpha}\left[\eta_{i j}\right]^{\beta}} \\
\eta_{i j}=1 / d_{i j}
\end{gathered}
$$

Where, $P_{i j}^{k}$ is the probability of $k^{t h}$ ant to choose node $j$ at a node $i, \tau_{i j}$ is the pheromone amount between nodes $i$ and $j, \eta_{i j}$ is the heuristic information between nodes $i$ and $j, d_{i j}$ is the path between nodes $i$ and $j$, and the parameters $\alpha$ and $\beta$ are the parameters to control the relative importance between the Pheromone amount and the heuristic information, respectively.

Step 4: Evaluate the cost function (ISE) as in Eq. (13) for each ant as follows:

$$
\operatorname{ISE}(k)=\int_{0}^{\infty}(Z-Y)^{2} d t
$$


Where; $k=1,2, \ldots, N ., Z$ is the desired input position of PMDC motor, and $Y$ is the actual output position of PMDC motor.

Step 5: Find the best node value, the minimum path cost to reach this node, which is the node that has a minimum (ISE) value among all the ants.

Step 6: Update the Pheromone trails with the evaporation process after all the ants have completed their tours as in equation (14).

$$
\Delta \tau_{i j}^{k}=\frac{L_{\min }}{L_{k}}
$$

Where, $\Delta \tau_{i j}{ }^{k}$ is the quantity of pheromone on each path, $L_{\min }$ is the best solution obtained by the set of the ants at the current iteration, and $L_{k}$ is the objective function value found by the ant $k$.

The total quantity of pheromones left by all ants between the nodes $i$ and $j$ are calculated by using equation (15):

$$
\Delta \tau_{i j}=\sum_{k=1}^{N} \Delta \tau_{i j}{ }^{k}
$$

Step 7: Next, the evaporation process occurs and the quantity of pheromones between all the nodes is updated as in equation (16):

$$
\tau_{i j}(t+1)=(1-\rho) \cdot \tau_{i j}(t)+\Delta \tau_{i j}
$$

Where, $0<\rho<1$ is the pheromone evaporation factor and $t$ is the current iteration.

Step 8: Stop if the maximum number of iterations (Iter) is reached. Otherwise, Step 3 to Step 8 is repeated.

\section{B. THE PSO ALGORITHM}

The PSO technique is proposed in 1995 by Russell Eberhart and James Kennedy. This algorithm is a heuristic global optimization method that mimics the social behavior of a colony or swarm of birds. Because of its high computational efficiency, this algorithm is commonly used in different engineering problems.

At first, each particle in the search space has an initially random position with an initial velocity equal to zero or small value. Such particles are moving in the search space in randomly direction and this movement of the particles is performed by evaluating them to show how near they are to the optimum solution by using a cost function. At each iteration each particle accelerates to the best location found by it so far $\left(p_{\text {best }}\right)$ and the global best position $\left(g_{\text {best }}\right)$ reached by any particle in the swarm [14]-[16]. The steps of PSO algorithm for tuning the LQR controller are given as follows:

Step 1: Initialize the algorithm parameters such as dimension of the problem ( $\mathrm{dim})$, population size $(N)$, maximum number of iterations (Iter), cognitive parameter $\left(c_{1}\right)$, social parameter $\left(c_{2}\right)$, initial random position for each bird $\left(X_{0}\right)$, zero initial velocity for each bird $\left(V_{0}\right)$, and inertia factor $(w)$.

Step 2: Set the best position for each particle ( $p_{\text {best }}$ ) equals its current position.

Step 3: Evaluate the cost function (ISE) as in Eq. (13) for each particle.

Step 4: Find the best position value $\left(g_{\text {best }}\right)$ which is a bird position that has a minimum (ISE) value among all the birds' positions.

Step 5: Update the velocity and position for each birds as follows:

$$
\begin{gathered}
V_{k m}^{t+1}=w V_{k m}^{t}+c_{1} r_{1}\left(p_{b e s t}^{t} t_{k m}^{t}-X_{k m}^{t}\right)+c_{2} r_{2}\left(g_{b e s t}^{t} t_{k m}^{t}-X_{k m}^{t}\right) \\
X_{k m}^{t+1}=X_{k m}^{t}+V_{k m}^{t+1}
\end{gathered}
$$


Where, $t=1,2, \ldots$, Iter $, k=1,2, \ldots, N, m=1,2, \ldots$, dim, and $r_{1}$ and $r_{2}$ are random mumbers between 0 and 1 .

Step 6: Evaluate again the cost function (ISE) for each particle as in Eq. (13). For each bird if its current cost function value is smaller than its previous value then the $\left(p_{\text {best }}\right)$ is changed with the current position.

Step 7: Find the smallest ( $\left.p_{\text {best }}\right)$ value among all birds and store it in $\left(g g_{\text {best }}\right)$ variable.

Step 8: If $\left(g g_{\text {best }}\right)$ value is smaller than $\left(g_{\text {best }}\right)$ then the $\left(g_{\text {best }}\right)$ value is replaced by the $\left(g g_{\text {best }}\right)$ value. Step 9: Stop if the maximum number of iterations (Iter) is reached. Otherwise, Step 5 to Step 9 is repeated.

\section{SIMULATION RESULTS AND DISCUSSIONS}

The PMDC motor specifications as shown in Table I are taken from [17]. The ACO and PSO tuning control algorithms for obtaining the optimal values of the weighting matrices of the LQR controller are performed by using the MATLAB simulation package to achieve the desired output position for the PMDC motor system as shown in Fig. 3. In the simulation results, a comparison is drawn from the results obtained between the LQR based ACO controller and LQR based PSO controller. The simulation results are presented to illustrate the performance and validity of these two design methods.

TABLE I. THE PARAMETERS OF THE PMDC MOTOR SYSTEM.

\begin{tabular}{cc}
\hline Description and Symbol & Value and Unit \\
\hline Armature Resistance $\left(R_{a}\right)$ & $5 \Omega$ \\
Coefficient of Viscous-Friction $\left(b_{m}\right)$ & $0.136 \mathrm{~N} . \mathrm{m} . \mathrm{s}$ \\
Moment of Inertia $\left(J_{m}\right)$ & $0.0025 \mathrm{Kg} \cdot \mathrm{m}^{2} / \mathrm{s}^{2}$ \\
Torque Constant $\left(K_{i}\right)$ & $0.245 \mathrm{~N} . \mathrm{m} / \mathrm{A}$ \\
Back EMF Constant $\left(K_{b}\right)$ & $0.245 \mathrm{~V} . \mathrm{s} / \mathrm{rad}$ \\
Armature Inductance $\left(L_{a}\right)$ & $0.01 \mathrm{H}$ \\
\hline
\end{tabular}

The states of the PMDC are $x_{1}, x_{2}$, and $x_{3}$ and represent the angular position $\left(\theta_{m}\right)$ in $(\mathrm{rad})$, the angular velocity $\left(w_{m}\right)$ in $(\mathrm{rad} / \mathrm{sec})$, and the armature current $\left(i_{a}\right)$ in $(\mathrm{amp})$, respectively. The openloop step response of the output position of the PMDC motor system is shown in Fig. 4. This Figure reveals that the system has an unstable response.

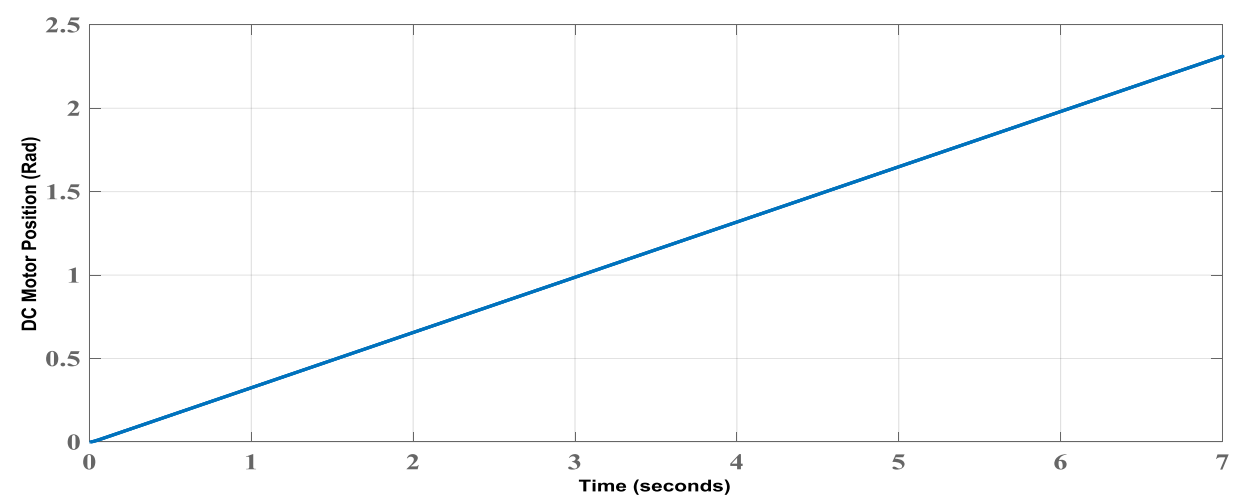

FIG. 4. STEP RESPONSE OF THE OPEN LOOP PMDC MOTOR SYSTEM. 
To investigate the optimal LQR controllers as shown in Fig. 3, the ACO and PSO tuning control methodologies are used to set the optimal elements of the weighting matrices of the LQR controller. These parameters lead to find the optimal control action and to minimize the position error with the minimum number of fitness evaluation, the parameters of the control methodology based on ACO and PSO algorithms are defined in Tables II and III, respectively.

TABLE II. THE PARAMETERS OF THE ACO ALGORITHM.

\begin{tabular}{cc}
\hline Description and Symbol & Value \\
\hline Population size $(N)$ & 25 \\
Maximum number of iteration (Iter) & 50 \\
Alpha $(\alpha)$ & 0.8 \\
Beta $(\beta)$ & 0.2 \\
Evaporation Rate $(\rho)$ & 0.7 \\
Number of Nodes for each Parameters \\
$\quad\left(n \_\right.$node) \\
Dimension of the Problem $($dim $)$
\end{tabular}

TABLE III. THE PARAMETERS OF THE PSO ALGORITHM.

\begin{tabular}{cc}
\hline Description and Symbol & Value \\
\hline Population size $(N)$ & 25 \\
Maximum number of iteration $($ Iter $)$ & 50 \\
Cognitive Parameter $\left(c_{1}\right)$ & 1.49618 \\
Social Parameter $\left(c_{2}\right)$ & 1.49618 \\
Inertia Factor $(w)$ & 0.7298 \\
Dimension of the Problem $(\mathrm{dim})$ & 4
\end{tabular}

The optimization ranges of LQR weighting matrices are set as in Table IV as follows:

TABLE IV. THE OPTIMIZATION RANGES OF LQR WEIGHTING MATRICES.

\begin{tabular}{ccc}
\hline Parameter & Min. & Max. \\
\hline$q_{11}$ & 0.001 & 12 \\
$q_{22}$ & 0.001 & 9 \\
$q_{33}$ & 0.001 & 3 \\
$R$ & 0.001 & 0.75 \\
\hline
\end{tabular}

The simulation results of the closed loop position control system with the variable step change as $(1,2$, and 3$)$ radian in the DC motor desired output position and LQR controllers with the initial output position of the system equals zero radian can be shown in Figs. 5 to 10. The optimal parameters of the controllers with the dynamic behavior of the system at each variable step change such as rising time, settling time, peak time, overshoots and steady state error are set as in Table V.

The output position response of the DC motor system in the variable step change using optimal LQR controllers has no overshoot in the transient state and at the steady-state, the error is equal to zero value in each step change, as shown in Fig. 5 


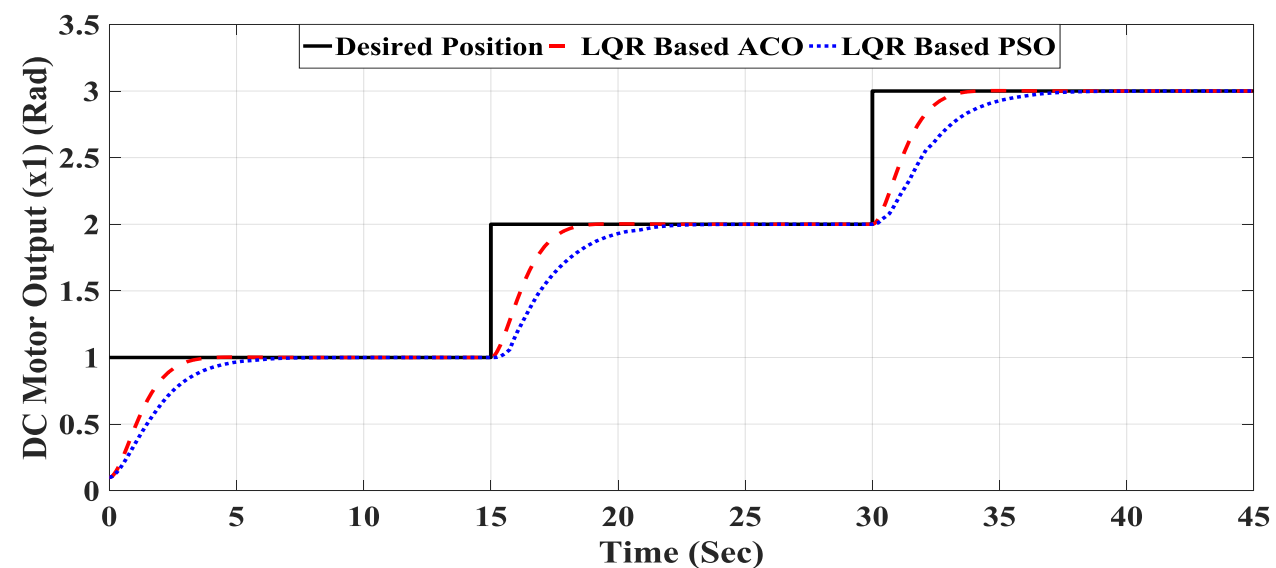

Fig. 5. ThE OUTPUT POSITION OF THE PMDC MOTOR.

TABLE V. THE OPTIMAL PARAMETERS OF THE CONTROLLERS WITH THE DYNAMIC BEHAVIOR OF THE SYSTEM

\begin{tabular}{ccc}
\hline Parameter & LQR based ACO & LQR based PSO \\
\hline$q_{11}$ & 7.0307 & 11.3201 \\
$q_{22}$ & 0.8494 & 8.4526 \\
$q_{33}$ & 2.1826 & 2.3845 \\
$R$ & 0.3646 & 0.4257 \\
$K_{1}$ & 4.3913 & 5.15672 \\
$K_{2}$ & 2.7196 & 4.28680 \\
$K_{3}$ & 2.3909 & 2.31100 \\
$T_{r}$ & $2.3993 \mathrm{sec}$ & $3.6824 \mathrm{sec}$ \\
$T_{p}$ & $0 \mathrm{sec}$ & $0 \mathrm{sec}$ \\
$T_{s}(2 \%$ error $)$ & $3.2773 \mathrm{sec}$ & $5.7728 \mathrm{sec}$ \\
$M p \%$ & 0 & 0 \\
$E_{\text {s.s }}$ & 0 & 0 \\
\hline
\end{tabular}

In Fig. 6, the position error signal of the closed loop PMDC motor system based on optimal LQR controllers was a small value in the transient and it has become zero at the steady state.

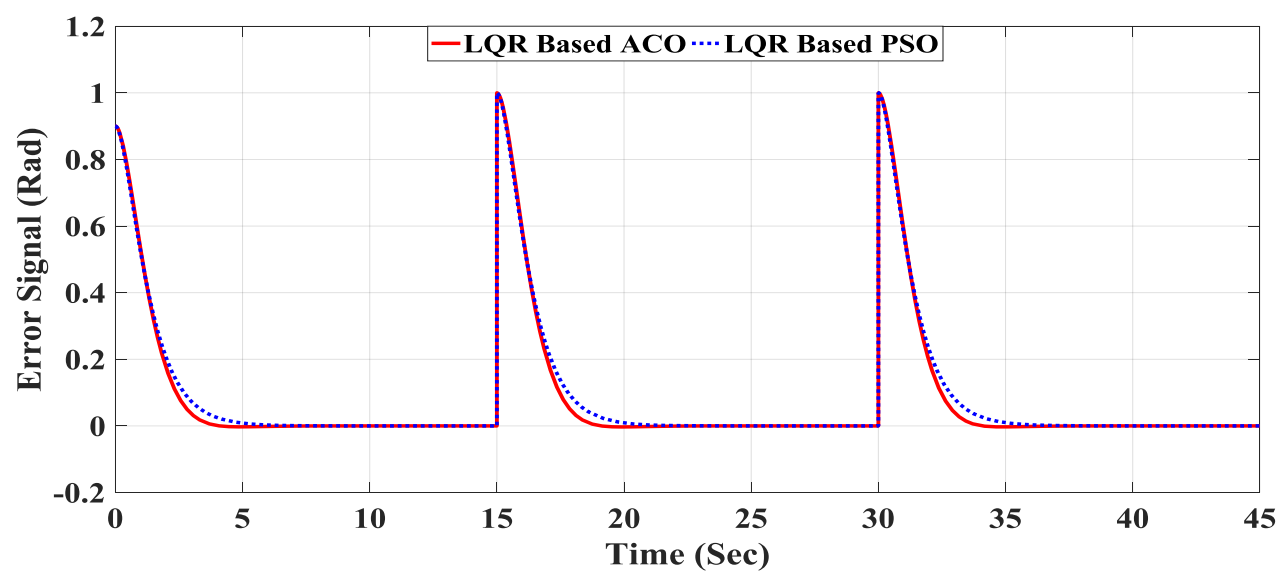

FIG. 6. THE POSITION ERROR SIGNAL. 
The control action response In Fig. 7 of the optimal LQR controllers was smooth without oscillation response, no spikes behavior and the action did not reach to the saturation state of 12 volt depending on the supply voltage of PMDC motor.

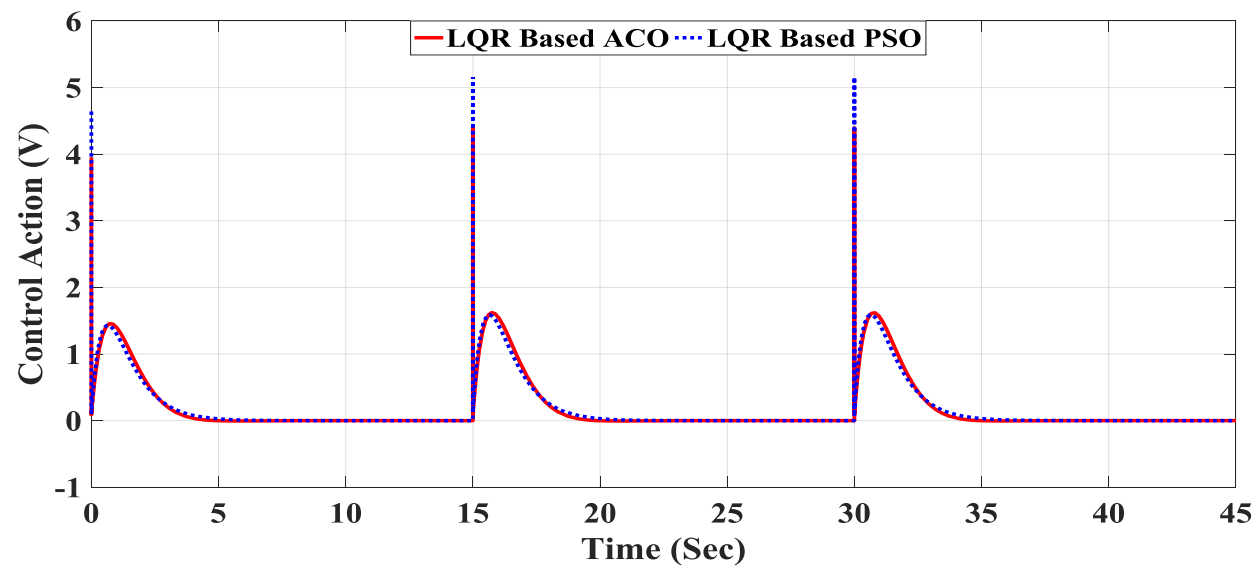

FIG. 7. THE CONTROL ACTION SIGNAL.

The improved cost function (ISE) of the PMDC motor model for the optimal LQR controllers at 50 iteration is clearly showed in Figs. 8 and 9.

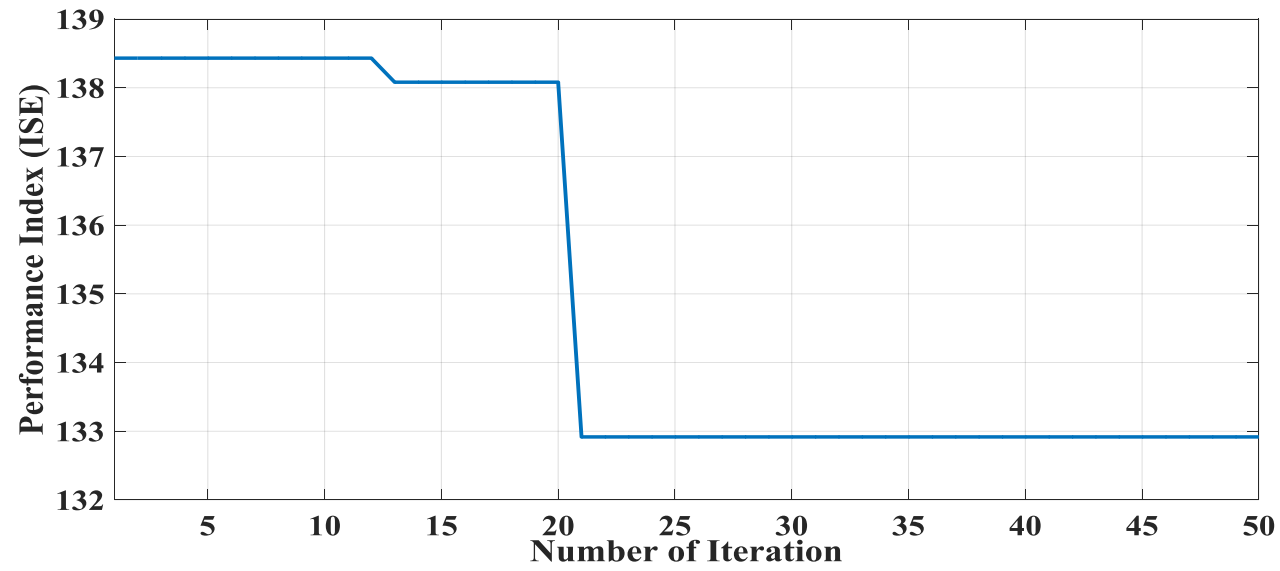

FIG. 8. THE PERFORMANCE INDEX (ISE) OF THE LQR BASED ACO ALGORITHM.

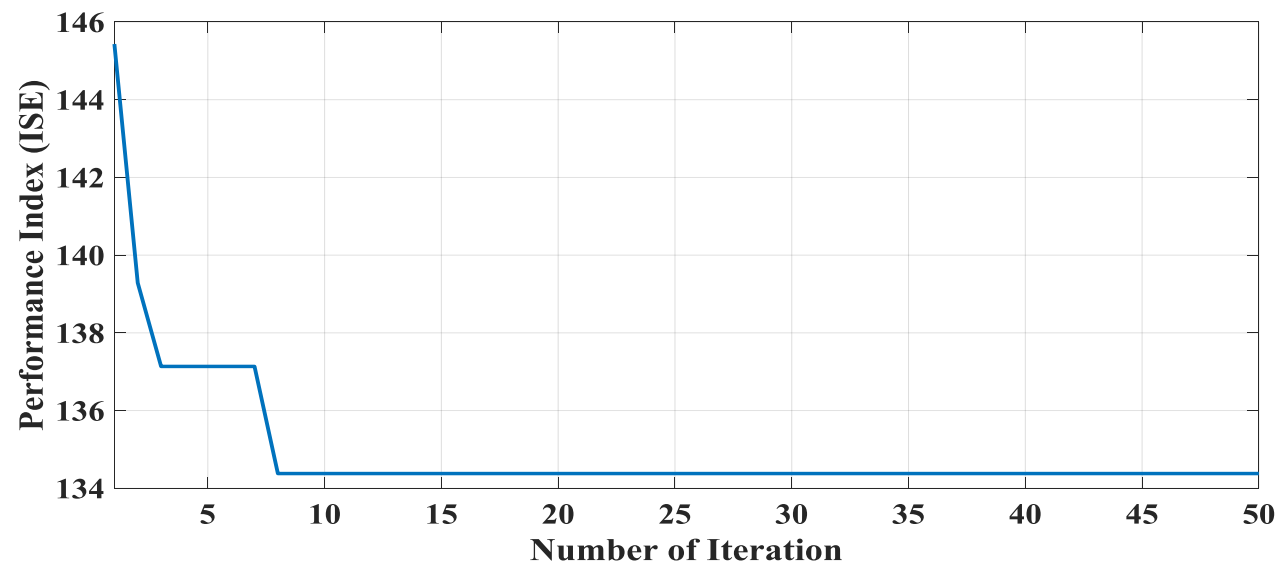

FIG. 9. THE PERFORMANCE INDEX (ISE) OF THE LQR BASED PSO ALGORITHM. 
The angular velocity $\left(x_{2}\right)$ state and the armature current $\left(x_{3}\right)$ state of the PMDC motor are shown in Figs. 10 and 11, respectively. These figures reveal that the angular velocity of the PMDC using LQR based ACO controller is faster than that of the PMDC using LQR based PSO controller and also consuming less current than that of the PMDC using LQR based PSO controller.

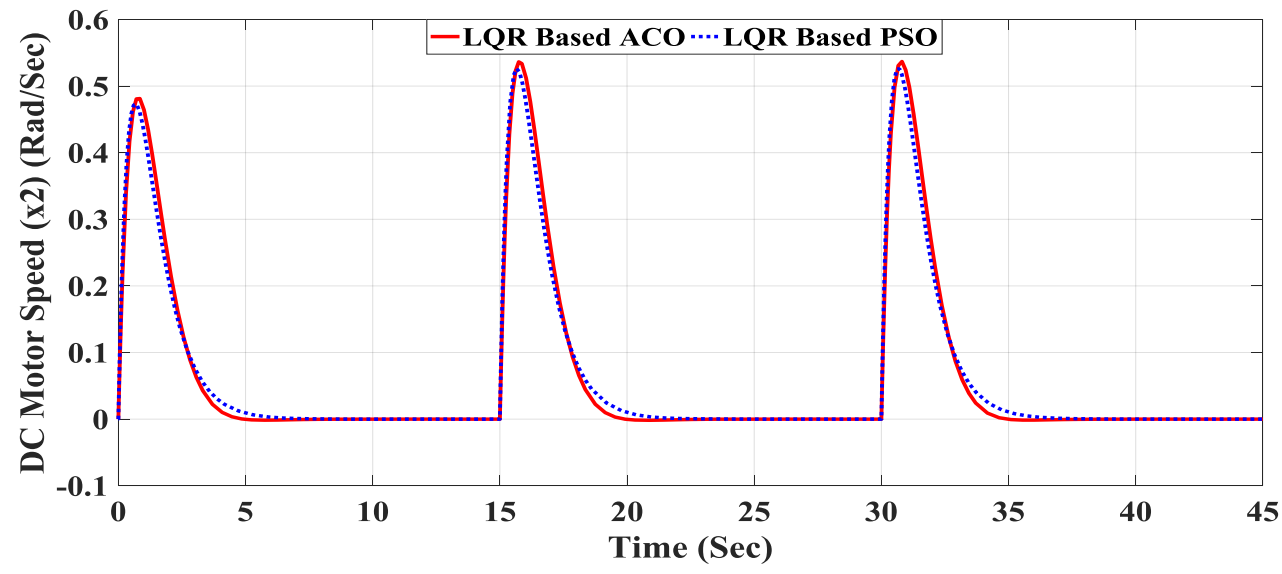

Fig. 10. THE ANGULAR VELOCITY OF THE PMDC MOTOR.

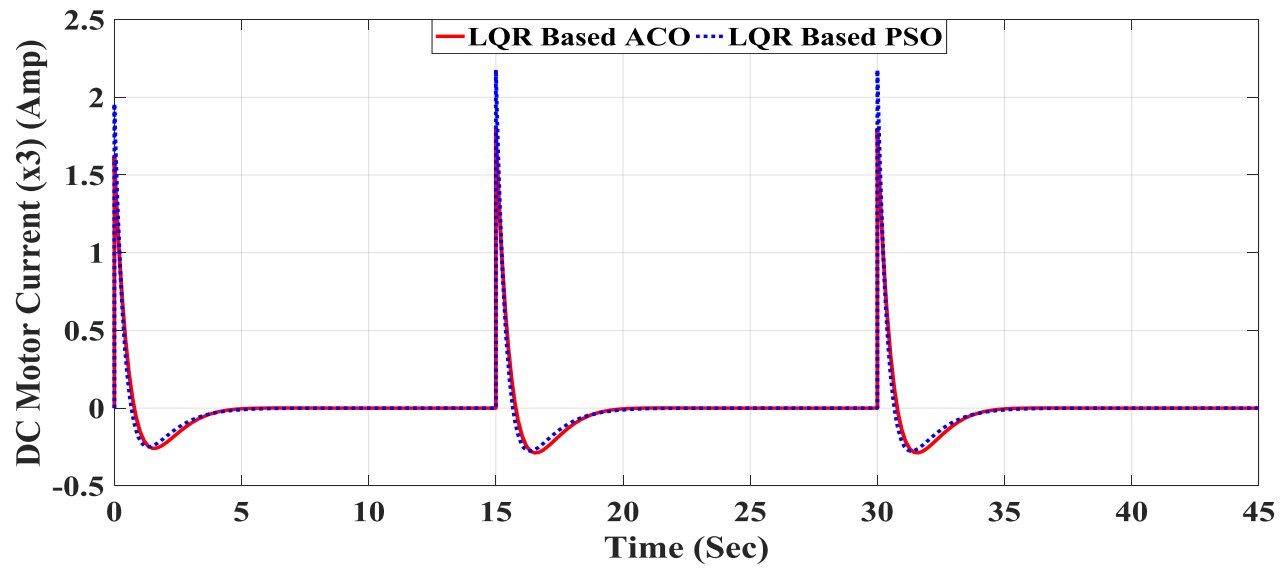

FIG. 11. THE CURRENT OF THE PMDC MOTOR.

To investigate the robustness performance of the LQR based ACO controller that has a better performance than the LQR based PSO controller according to the simulation results, an external torque disturbance $\left(T_{d}\right)$ which equals $(1 \mathrm{~N} . \mathrm{m})$ is added at the moment $(22-23 \mathrm{sec})$. The output position response of the PMDC motor has a small overshoot at $(22 \mathrm{sec}$ ) during adding disturbance and the error equals zero value at the steady state response as shown in Fig. 12. 


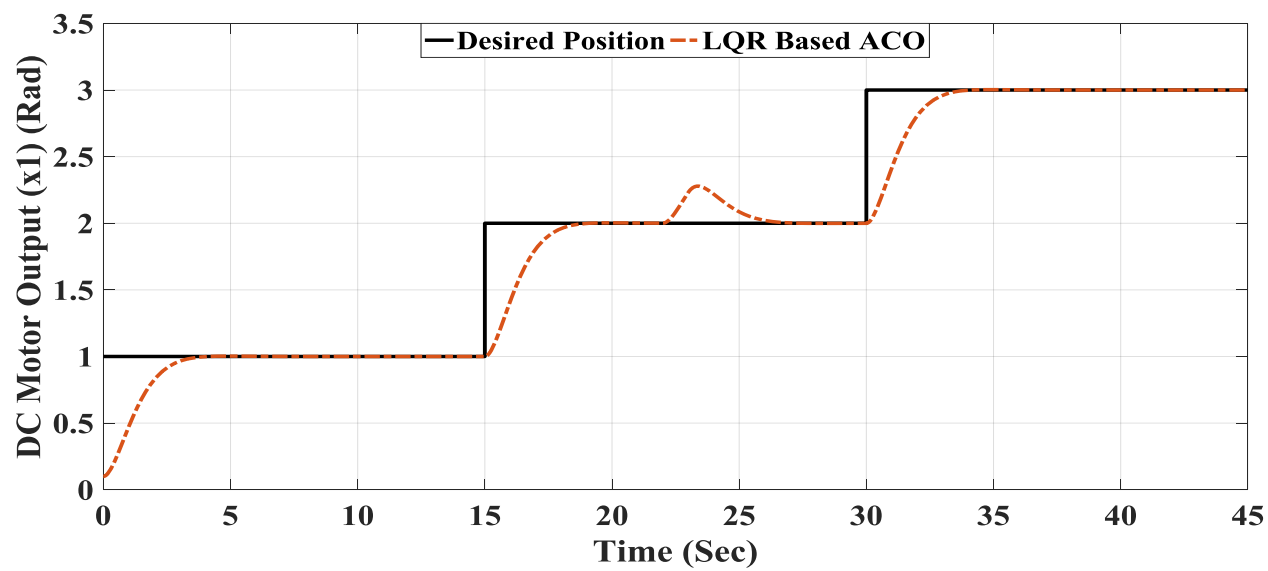

Fig. 12. THE OUTPUT POSITION OF THE PMDC MOTOR UNDER DISTURBANCE.

The small value of the error position signal between the desired position and the output position of the PMDC motor system is shown in Fig. 13, the error has a small value at the transient state and in the steady state, the error becomes very close to zero value.

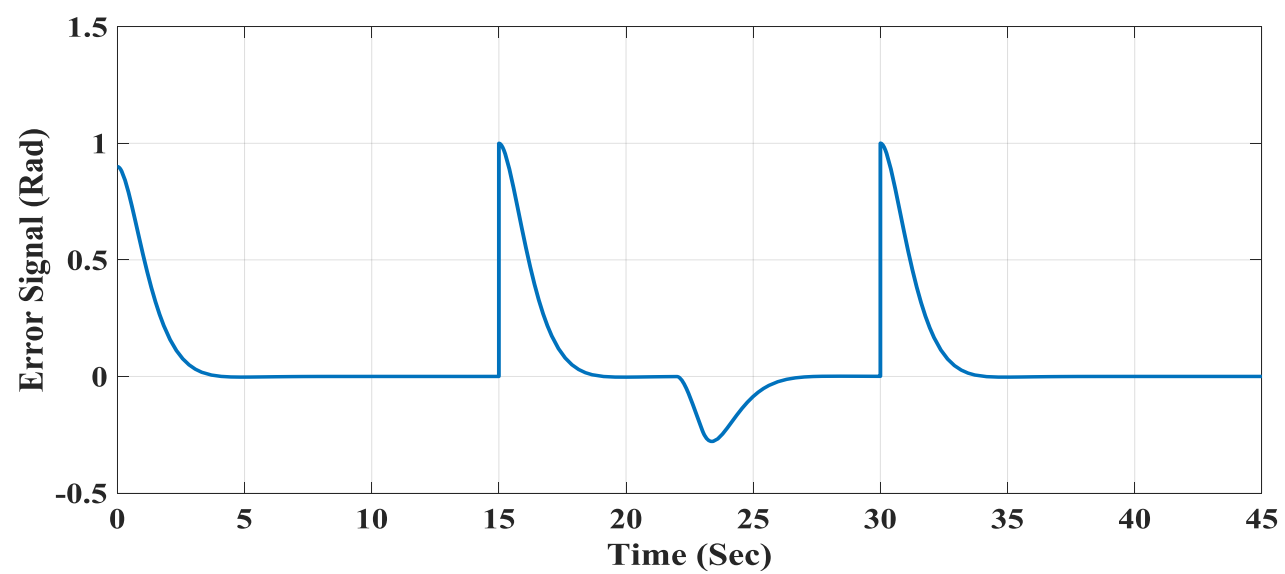

Fig. 13. THE POSITION ERROR SIGNAL UNDER DISTURBANCE.

The response of the control action is shown in Fig. 14, which has a capability to track the error position signal of the PMDC motor system to follow the desired position as step change and reduce the effect of the torque external disturbance.

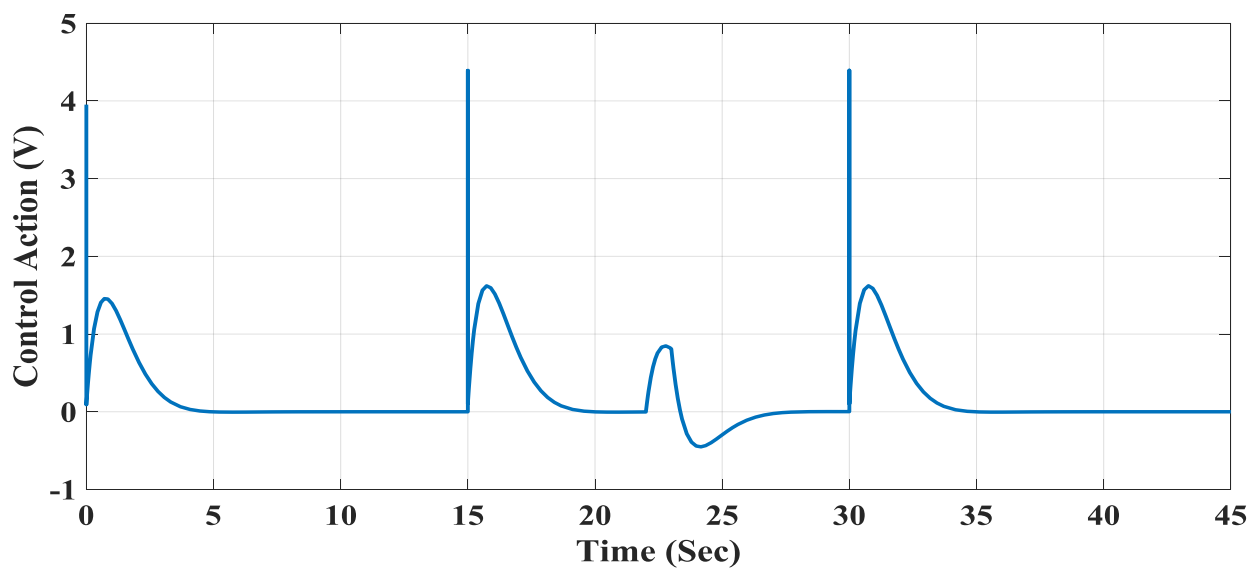

FIG. 14. THE CONTROL ACTION SIGNAL UNDER DISTURBANCE. 
Finally, the angular velocity $\left(x_{2}\right)$ state and the armature current $\left(x_{3}\right)$ state of the PMDC motor are shown in Figs. 15 and 16, respectively.

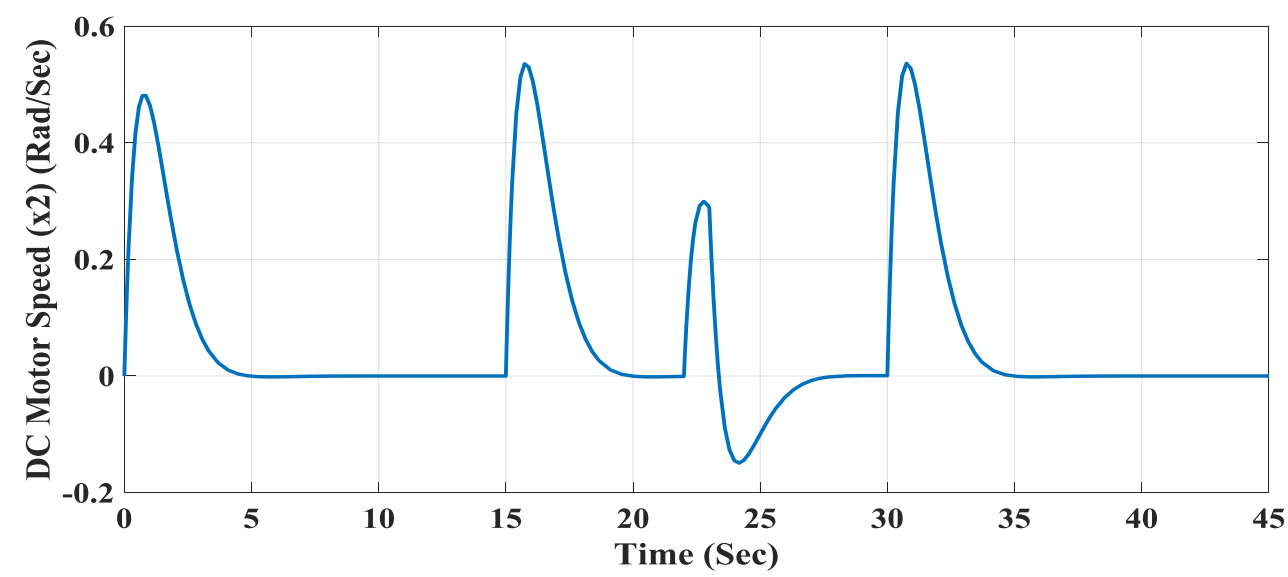

FIG. 15. THE ANGULAR VELOCITY OF THE PMDC MOTOR UNDER DISTURBANCE.

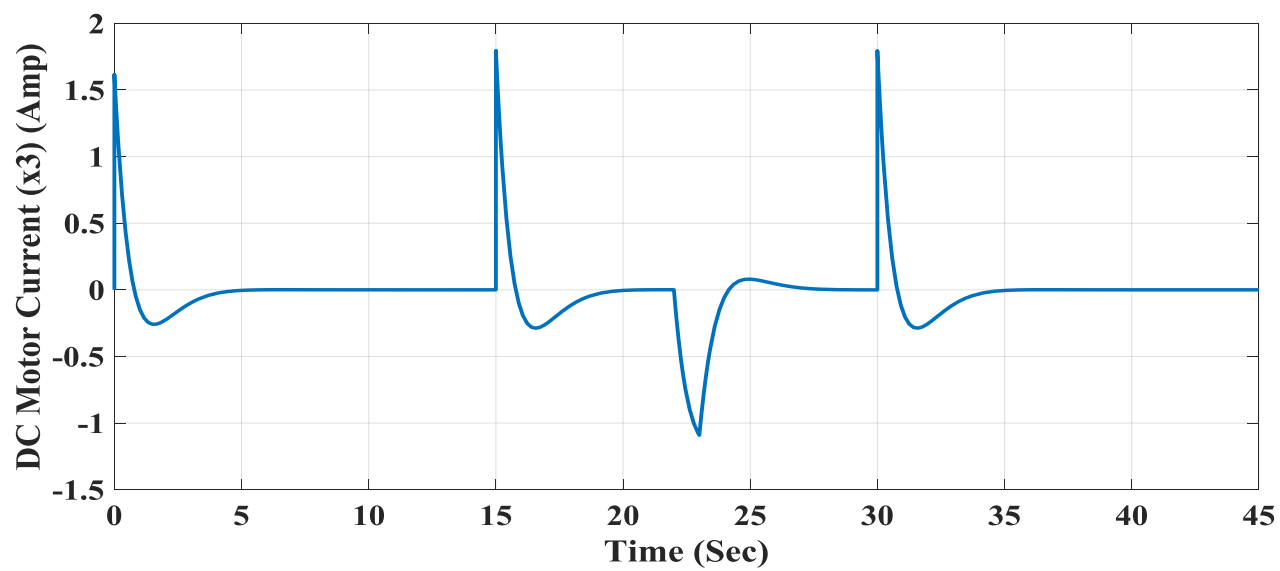

FIG. 16. THE CURRENT OF THE PMDC MOTOR UNDER DISTURBANCE.

\section{CONCLUSIONS}

In this work, a position control of PMDC motor using different methodologies for tuning the weighting matrices of the LQR controller is successfully designed and constructed using MATLAB Package. According to the obtained simulation results the following points are concluded as listed below:

- The ACO algorithm has the ability to fast pick up the optimal weighting matrices of the LQR controller with the minimum number of fitness evaluation in comparison with the PSO algorithm.

- A proper voltage control action was obtained without a saturation voltage state.

- Excellent tracking performance was achieved by changing the desired output position level.

- The performance of the LQR based ACO controller is better than that of the LQR based PSO controller in terms of obtaining desirable time response specifications and minimum unsaturated state voltage control law that will stabilize the PMDC motor system performance and minimize the position tracking error of the system output. Furthermore, the cost function is decreased.

- High robustness performance was obtained by adding an external torque as a disturbance effect to PMDC motor system using LQR based ACO controller. 


\section{REFERENCES}

[1] K. Hassani and W-S. Lee, "Optimal Tuning of Linear Quadratic Regulators Using Quantum Particle Swarm Optimization," Proceedings of the International Conference of Control, Dynamic Systems, and Robotics, Ottawa, Ontario, Canada, May 15-16, 2014, paper No. 59, pp. 1-8.

[2] H.I. Ali and A. A. V Mahmood, "Linear Quadratic Regulator Design using Particle Swarm Optimization," The islamic college university journal, Najaf, Vol. 0, Issue 38, 2016, pp. 159-165.

[3] T. Marada, R. Matousek, and D. Zuth, "Design of Linear Quadratic Regulator (LQR) Based on Genetic Algorithm for Inverted Pendulum," MENDEL — Soft Computing Journal, Vol. 23, No. 1, 2017, P.P. 149- 156.

[4] A. Al-Mahturi and H. "Wahid, Optimal Tuning of Linear Quadratic Regulator Controller Using a Particle Swarm Optimization for Two-Rotor Aerodynamical System," World Academy of Science, Engineering and Technology, International Journal of Electronics and Communication Engineering, Vol. 11, No. 2, 2017, pp. 196-202.

[5] T. ABUT, and M. Huseyinoglu, "Modeling and Optimal Trajectory Tracking Control of Wheeled a Mobile Robot," Caucasian Journal of Science, Vol. 6, Issue 2, 2019, P.P. 137- 146.

[6] U. Önen, A. Çakan, and I. İlhan, "Performance comparison of optimization algorithms in LQR controller design for a nonlinear system," Turkish Journal of Electrical Engineering \& Computer Sciences, Vol. 27, No. 3, 2019, P.P. 1938- 1953.

[7] A. A. Mahfouz, A. A. Aly and F. A. Salem, Mechatronics Design of a Mobile Robot System, I.J. Intelligent Systems and Applications, Vol. 5, No. 3, Feb. 2013, pp. 23 - 36.

[8] T.S. Abd El Ghaffar, A.S. El-Wakeel, A. Eliwa and R.M. Mostafa, Optimal Position Control of Permanent Magnet DC Motor (PMDC), 14th International Conference on AEROSPACE SCIENCES \& AVIATION TECHNOLOGY, ASAT -14 - May 24 - 26, 2011.

[9] A. S. Al-Araji, Design of Nonlinear PID Neural Controller for the Speed Control of a Permanent Magnet DC Motor Model based on Optimization Algorithm, Al- Khwarizmi Engineering Journal, Vol. 10, No. 1, 2014, pp. 72-82.

[10] K. Ogata, “Modern Control Engineering,” Pearson Education, Inc., 5th Edition, New Jersey, 2010.

[11] M. Dorigo and T. Stützle, “Ant Colony Optimization," Massachusetts Institute of Technology, London, England, 2004.

[12] A. Jacknoon and M. A. Abido, "Ant Colony Based LQR and PID tuned Parameters for Controlling Inverted Pendulum," International Conference on Communication, Control, Computing and Electronics Engineering (ICCCCEE), Khartoum, Sudan, 16-18 Jan.2017.

[13] A. M. Hamza, M. S. Saad, H. M. Rashad and A. Bahgat, "Gas Turbine LQR, Integral Controllers and Optimal PID Tuning by Ant Colony Optimization Comparative Study," International Journal of Computer Science and Telecommunications, Vol. 4, Issue 1, January, 2013, pp. 35-41.

[14] D. P. Rini, S. M. Shamsuddin and S. S. Yuhaniz, "Particle Swarm Optimization: Technique, System and Challenges", International Journal of Computer Applications, Vol. 14, No. 1, Jun. 2011, pp. 19 - 27.

[15] S. Talukder, "Mathematical Modelling and Applications of Particle Swarm Optimization", M.Sc. Thesis in Mathematical Modeling and Simulation, School of Engineering at Blekinge Institute of Technology, Karlskrona, Sweden, Feb. 2011

[16] C. Li, "Particle Swarm Optimization in Stationary and Dynamic Environments", PhD Thesis in department of Computer Science, University of Leicester, England, Dec. 2010.

[17] M. A. S. Mohammed, A. J. Humaidi and A. A. Al jodah, "Design and Simulation of L1-Adaptive Controller for Position Control of DC Servomotor," Al-Khwarizmi Engineering Journal, Vol. 12, No. 2, 2016, P.P. 100- 114. 